\title{
Clinical and pathologic factors associated with development of hepatocellular carcinoma in patients with hepatitis virus-related cirrhosis: a long-term follow-up study
} Vanessa Rosas-Camargo 1,4, José Luis Rodríguez-Díaz ${ }^{1,4}$, Olynka Vega-Vega², Aniela Méndez-Reguera1,4, Daniela Morales Espinosa ${ }^{1,4}$, Jorge Luis MartínezTlahuel ${ }^{1}$, Armando Gamboa-Domínguez ${ }^{3}$ and Oscar Arrieta*1,4

\begin{abstract}
Address: ${ }^{1}$ Department of Medical Oncology, Instituto Nacional de Cancerología, Mexico City, Mexico, ${ }^{2}$ Department of Internal Medicine, Instituto Nacional de Ciencias Médicas y Nutrición, Mexico City, Mexico, ${ }^{3}$ Department of Pathology, Instituto Nacional de Ciencias Médicas y Nutrición, Mexico City, Mexico and ${ }^{4}$ Universidad Nacional Autónoma de México, Mexico City, Mexico
\end{abstract}

Email: Oscar Arrieta* - ogar@servidor.unam.mx

* Corresponding author

from 24th Annual Meeting of the National Cancer Institute of Mexico

Mexico City, Mexico. 14-17 February 2007

Published: 5 February 2007

BMC Cancer 2007, 7(Suppl I):A33 doi:I0.I 186/I47|-2407-7-SI-A33

This article is available from: http://www.biomedcentral.com//47I-2407/7/SI/A33

(c) 2007 Rosas-Camargo et al; licensee BioMed Central Ltd.

\section{Background}

Hepatocellular carcinoma (HCC) represents $>90 \%$ of primary liver neoplasms and develops mainly in patients with liver cirrhosis. Risk factor identification for development of HCC in patients with cirrhosis possesses great clinical relevance due to its high incidence and poor prognosis when detected at advanced stages. The aim of our study was to identify HCC development-associated risk factors in a cohort of patients with hepatitis virus-related chronic liver disease and cirrhosis.

\section{Materials and methods}

Patients with a diagnosis of hepatitis virus-related cirrhosis from January 1980 to January 2000 were included. Patients were followed with abdominal ultrasound and determination of alpha-fetoprotein levels, physical examination, and routine biochemical tests every 3-6 months. The endpoint in this study was defined as development of HCC. Liver histology was evaluated according to the French METAVIR Cooperative Study Group (METAVIR) score.

\section{Results}

Two hundred and eighty two patients met the inclusion criteria; the majority of these ( $86 \%$ ) had a serologic diagnosis of hepatitis C virus, and only $14 \%$ had hepatitis B virus at the time of diagnosis of cirrhosis, while 56 and $37 \%$ were classified as Child A and B, respectively, and only $7 \%$ as Child C. Histological activity was mild in $59 \%$ of patients, and moderate and severe in $41 \%$. Mean annual incidence was $1.87 \%$, and 22 and $35 \%$ of patients developed HCC at 10 and 15 years of follow-up, respectively. Diagnosis of HCC was made by histopathology in $37 \%$ and by tumoral lesion-associated alpha-fetoprotein elevation confirmed by imaging studies in $63 \%$. In multivariate analysis, we found three variables associated with HCC: moderate to severe histological activity; platelet count $<105 \times 10^{3} / \mathrm{mm}^{3}$, and alpha-fetoprotein $>5 \mathrm{ng} / \mathrm{mL}$ (see Table 1). We divided patients into two groups according to regression coefficient: low and high-risk; patients assigned to the low-risk group showed 5-, 10-, and 15-year HCC incidences of 3.4, 6.4, and 6.4\%, respectively, in contrast to patients from the high-risk group, who showed incidences of $17.8,33.5$, and $56.8 \%$, respectively.

\section{Conclusion}

We found three HCC-associated variables: histological activity; platelet count and alpha-fetoprotein levels. Patients with high risk for developing hepatocellular carcinoma must be considered candidates for closer followup. 
Table I: Variables with independent predictive value for HCC in the multivariate analysis

\begin{tabular}{lccc}
\hline Variables & Hazard ratio $(95 \%$ confidence interval) & Coefficients & $P$ value (log rank) \\
\hline Platelet count & $3.7(1.2-11)$ & 1.3 & 0.025 \\
$\alpha$-fetoprotein & $5.41(1.86-15.7)$ & 1.23 & 0.02 \\
Histological activity & $7.6(1.7-33)$ & 2.03 & 0.007 \\
\hline
\end{tabular}

